\title{
Partial Stability of Conformable Stochastic Systems
}

\author{
Abdellatif Ben Makhlouf $\mathbb{D I}^{1}$ and Lassaad Mchiri $\mathbb{D D}^{2}$ \\ ${ }^{1}$ Mathematics Department, College of Science, Jouf University, P.O. Box: 2014, Sakaka, Saudi Arabia \\ ${ }^{2}$ Department of Statistics and Operations Research, College of Sciences, King Saud University, Riyadh 11451, Saudi Arabia \\ Correspondence should be addressed to Abdellatif Ben Makhlouf; abmakhlouf@ju.edu.sa
}

Received 26 October 2021; Revised 7 December 2021; Accepted 1 February 2022; Published 21 February 2022

Academic Editor: Quanxin Zhu

Copyright (c) 2022 Abdellatif Ben Makhlouf and Lassaad Mchiri. This is an open access article distributed under the Creative Commons Attribution License, which permits unrestricted use, distribution, and reproduction in any medium, provided the original work is properly cited.

\begin{abstract}
In this study, we investigate the almost sure exponential stability (ASES) and the exponential stability in the p-th moment (ESPM) with respect to part of the variable of conformable stochastic systems (CSSs) by using the Lyapunov methods and the stochastic calculus techniques. In the last section, we illustrate our results with a theoretical example.
\end{abstract}

\section{Introduction}

Many deterministic phenomena were described by the differential equations. Taking into account the random phenomena, formally one must take into account the "stochastic perturbed term," which transforms the equations into stochastic differential equations (SDEs).

The Caputo derivative and Riemann-Liouville derivative do not verify the chain and Leibniz rules, which sometimes prevent us from applying these derivatives to some physical problem with the standard Newton derivative. Recently, in [1], the authors invented the new fractional derivative called "conformable integral and fractional derivatives." This well-known derivative satisfies the chain and Leibniz rules.

In fact, according to the special characteristics of the fractional derivative (which is different from the property of the classical derivative), the compatibility of the stochastic integral and fractional integral encounters many difficulties. Fractional integrals contain singular integral terms, which makes it difficult to calculate its numerical solution and estimate its asymptotic solution. Taking into account all the considerations, we present conformable stochastic differential equations.

In particular, the asymptotic behaviour and the stability theory of the solution of ordinary stochastic systems, conformable fractional-order stochastic systems, and deterministic systems were investigated by many researchers (see [2-6]).

In the last decades, it has established its effectiveness as an essential tool in many real-world applications, such as physics [7], biology [4, 8, 9], and control theory $[2,3,5,10-14]$. In fact, Neirameh in [4] proposed a new method to find exact solutions of the general biological population model. By using the trial solution algorithm and the complete discrimination system, Odabasi in [9] has proved the existence of the exact solutions of the biological population model. Using the fixed-point method, Das in [2] showed the controllability and the existence of mild solution for a class of nonlinear systems. On the other hand, Younus et al. in [14] investigated the observability of linear timeinvariant control systems.

In the last years, the stability analysis of conformable systems was investigated by many researchers. In [2, 3, 12], the authors studied the stability of the solution of conformable deterministic systems. In the literature, there is a few work about the stability of the solution of conformable stochastic systems (see $[5,6])$. On the other hand, in many real-world phenomena, such stability is sometimes too difficult to be satisfied. Consequently, the concept of partial stability (see [15-20]) has been presented, and the Lyapunov technique, as an important tool, has been utilized to study the stability with respect to part of the variable in various real-world important phenomena. To the best of our 
knowledge, there is no existing paper on the stability with respect to part of the variables of CSSs. In $[17,19]$, the authors studied the partial asymptotic stability in the probability of ordinary stochastic differential equations by using the Lyapunov methods. In our paper, we will extend the results in $[17,19]$ to the case of nonlinear conformable systems and we will investigate the ASES and the ESPM with respect to part of the variable.

In our paper, we investigate the partial ESPM and the partial ASES. In this sense, our results present a complete generalization of the work [17].

The main contributions of our paper are as follows:

(i) Study the partial ASES and the partial ESPM of CSSs under some new criteria

(ii) Different from the previous results in [17], taking into account the influence of the conformable fractional derivative in the partial stability theory make our results more general

This paper is organized as follows. In Section 2, we recall some basic notions. In Section 3, we investigate the partial ESPM and the partial ASES of CSSs. In Section 4, we study the exponential instability in the p-th moment with respect to all variables of CSSs. In Section 5, we illustrate our theory with an example.

\section{Preliminaries}

In this section, some basic results are presented.

The complete probability space is denoted by $\left\{\Omega, \mathbb{F},\left(\mathbb{F}_{\delta}\right)_{\delta \geq 0}, P\right\}$, where $\left\{\mathbb{F}_{\delta}\right\}_{\delta \geq 0}$ is a filtration satisfying the usual conditions. $W(\delta)$ is an $m$-dimensional Brownian motion defined on $\left\{\Omega, \mathbb{F},\left(\mathbb{F}_{\delta}\right)_{\delta \geq 0}, P\right\}$. The set of $\mathbb{R}^{n}$-valued $\mathbb{F}_{\delta}$-adapted processes $\{\psi(\delta)\}_{t \leq \delta \leq s}$ is denoted by $\mathscr{L}^{2}([t, s]$, $\left.\mathbb{R}^{n}\right)$ such that $\int_{t}^{s}|\psi(\delta)|^{2} \mathrm{~d} \delta<\infty$ a.s. Let $\mathscr{C}\left([-\gamma, 0] ; \mathbb{R}^{n}\right)$ denote the set of functions $\psi$ from $[-\gamma, 0]$ to $\mathbb{R}^{n}$ that are rightcontinuous and have limits on the left. $\mathscr{C}\left([-\gamma, 0] ; \mathbb{R}^{n}\right)$ is equipped with the norm $\|\psi\|=\sup _{-\gamma \leq \delta \leq 0}|\psi(\delta)|$ and $|m|=\sqrt{m^{T} m}$ for any $m \in \mathbb{R}^{n}$. The set of all $\mathbb{F}_{0}$-measurable bounded $\mathscr{C}\left([-\gamma, 0] ; \mathbb{R}^{n}\right)$-valued random variables $\nu=\{\nu(\kappa):-\gamma \leq \kappa \leq 0\}$ are denoted by $\mathscr{C}_{\mathbb{F}_{0}}^{b}\left([-\gamma, 0] ; \mathbb{R}^{n}\right)$. Let $L_{\mathbb{F}_{\delta}}^{2}\left([-\gamma, 0] ; \mathbb{R}^{n}\right), \delta \geq 0$, denote the set of all $\mathbb{F}_{\delta}$-measurable, $\mathscr{C}\left([-\gamma, 0] ; \mathbb{R}^{n}\right)$-valued random variables $\varpi=\{\varpi(\kappa):-\gamma \leq \kappa \leq 0\}$ satisfies $\sup _{-\gamma \leq \kappa \leq 0} E|\oplus(\kappa)|^{2}<\infty$.

Definition 1 [21]. The conformable derivative (CD) of a function $G:[\Theta, \infty) \longrightarrow \mathbb{R}$ is defined by

$$
T_{\Theta}^{\alpha} G(\delta)=\lim _{d \longrightarrow 0} \frac{G\left(\delta+d(\delta-\Theta)^{1-\alpha}\right)-G(\delta)}{d},
$$

for all $\delta>\Theta, \alpha \in(0,1)$, while

$$
T_{\Theta}^{\alpha} G(\Theta)=\lim _{d \longrightarrow 0}+T_{\Theta}^{\alpha} G(\delta) .
$$

Definition 2 [21]. The conformable integral (CI) of a function $G:[\Theta, \infty) \longrightarrow \mathbb{R}$ is defined by

$$
I_{\Theta}^{\alpha} G(\delta)=\int_{\Theta}^{\delta}(\xi-\Theta)^{\alpha-1} G(\xi) \mathrm{d} \xi,
$$

for all $\delta>\Theta, \alpha \in(0,1)$.

Lemma 1 [21]. Let $G \in C([\Theta, \infty), \mathbb{R})$ and $0<\alpha<1$. Then, $\forall \delta>\Theta$, we obtain

$$
T_{\Theta}^{\alpha} I_{\Theta}^{\alpha} G(\delta)=G(\delta) .
$$

Lemma 2 [22]. Let $\alpha \in(0,1), v_{1}, v_{2}, \varsigma, v \in \mathbb{R}$, and the functions $G_{1}, G_{2}:[\Theta,+\infty) \longrightarrow \mathbb{R}$ such that $T_{\Theta}^{\alpha} G_{i}(\delta)$ exists on $(\Theta,+\infty)$ for $i=1,2$. Then,

(i) $T_{\Theta}^{\alpha}\left(v_{1} G_{1}+v_{2} G_{2}\right)=v_{1} T_{\Theta}^{\alpha} G_{1}+v_{2} T_{\Theta}^{\alpha} G_{2}$

(ii) $T_{\Theta}^{\alpha} v=0$

(iii) $T_{\Theta}^{\alpha}\left(G_{1} G_{2}\right)=G_{1} T_{\Theta}^{\alpha} G_{2}+G_{2} T_{\Theta}^{\alpha} G_{1}$

Remark 1. Let $G:[\Theta, \infty) \longrightarrow \mathbb{R}^{n}$ such that $T_{\Theta}^{\alpha} G(\delta)$ exists on $(\Theta, \infty)$. Then, $T_{\Theta}^{\alpha} G^{T} G(\delta)$ exists on $(\Theta, \infty)$ and

$$
T_{\Theta}^{\alpha} G^{T} G(\delta)=2 G(\delta)^{T} T_{\Theta}^{\alpha} G(\delta), \quad \forall \delta>a .
$$

Definition 3 [3]. The conformable exponential function is defined by

$$
\Xi_{\alpha}(\nu, \delta)=\exp \left(\nu \frac{\delta^{\alpha}}{\alpha}\right)
$$

where $\alpha \in(0,1)$ and $\nu \in \mathbb{R}$.

Remark 2. Since the conformable derivative has no spatial characteristic for the fractional derivative, we can directly explicit the solution of the system, evaluate the numerical solution, and approximate the error of the asymptotic solution.

\section{Main Results}

Consider the following CSS on $\left[\delta_{0}, \infty\right)$, for any $\chi \in(1 / 2,1)$ :

$$
\left\{\begin{array}{l}
T_{\delta_{0}}^{\chi} y=h_{\delta_{0}}(\delta, y)+\phi_{\delta_{0}}(\delta, y) \frac{d W(\delta)}{d \delta}, \\
y\left(\delta_{0}\right)=y_{0},
\end{array}\right.
$$

where the initial condition $y_{0}=\left(y_{01}, y_{02}\right)^{T} \in \mathbb{R}^{d} \times \mathbb{R}^{k}$, with $d+k=n$, which is independent of $W(\cdot)$, $y=\left(y_{1}, y_{2}\right)^{T} \in \mathbb{R}^{d} \times \mathbb{R}^{k}, \quad h_{\delta_{0}}(\cdot, \cdot):\left[\delta_{0}, \infty\right) \times \mathbb{R}^{n} \longrightarrow \mathbb{R}^{n}$, $\delta_{0}>0$, and $\phi_{\delta_{0}}(\cdot, \cdot):\left[\delta_{0}, \infty\right) \times \mathbb{R}^{n} \longrightarrow \mathbb{R}^{n}$ are continuous. Suppose that $h_{\delta_{0}}$ and $\phi_{\delta_{0}}$ are smooth enough, i.e., satisfying the Lipschitz and the growth condition to ensure the existence and the uniqueness of a global solution of system (7) (for more details, see Theorem 4.3 in [6]). 
Definition 4. We call that an $\mathbb{R}^{n}$-valued stochastic process $y(\cdot)$ is a solution of $(7)$ if $y(\delta)$ is continuous, $\mathbb{F}_{\delta}$-adapted, and

$$
y(\delta)=y_{0}+\int_{\delta_{0}}^{\delta} h_{\delta_{0}}(l, y(l))\left(l-\delta_{0}\right)^{\chi-1} \mathrm{~d} l+\int_{\delta_{0}}^{\delta} \phi_{\delta_{0}}(l, y(l))\left(l-\delta_{0}\right)^{\chi-1} \mathrm{~d} W(l), \quad \delta \in\left[\delta_{0}, \infty\right) .
$$

Definition 5. Let $p>0$. System (7) is said to be $y_{1}$-ASES, if

$$
E\left|y_{1}(\delta)\right|^{p} \leq \xi_{1}\left|y\left(\delta_{0}\right)\right|^{p} \Xi_{\chi}\left(-\xi_{2},\left(\delta-\delta_{0}\right)\right), \quad \forall \delta \geq \delta_{0} \geq 0 .
$$
there exist positive constants $c_{1}$ and $c_{2}$ satisfying, for any $y\left(\delta_{0}\right) \in \mathbb{R}^{n}$,

$$
\left|y_{1}(\delta)\right|^{p} \leq c_{1}\left|y\left(\delta_{0}\right)\right|^{p} \Xi_{\chi}\left(-c_{2},\left(\delta-\delta_{0}\right)\right), \quad \forall \delta \geq \delta_{0} \geq 0 .
$$

Definition 6. [16]. Let $p>0$. System (7) is said to be $y_{1}$-ESPM, if there exist positive constants $\xi_{1}$ and $\xi_{2}$ satisfying, for any $y\left(\delta_{0}\right) \in \mathbb{R}^{n}$,

Remark 3. Note that, in Definition 7, when $p=2$, system (7) is said to be $y_{1}$-exponentially stable in mean square $\left(y_{1}\right.$-ESMS $)$.

Let $\mathscr{W}(\delta, y) \in C^{1,2}\left(\left[\delta_{0},+\infty\left[\times \mathbb{R}^{n}, \mathbb{R}_{+}\right)\right.\right.$. By Itô formula (see [6]), one has the following: for $\delta>\delta_{0}$,

$$
\begin{aligned}
T_{\delta_{0}}^{\chi} \mathscr{W}(\delta, y) & =\mathscr{L}_{\chi}^{\delta_{0}} \mathscr{W}(\delta, y)+\mathscr{W}_{y}(\delta, y) \phi_{\delta_{0}}(\delta, y) \frac{\mathrm{d} W(\delta)}{\mathrm{d} \delta}, \\
\mathrm{d} \mathscr{W}(\delta, y) & =\mathscr{L}_{\chi}^{\delta_{0}} \mathscr{W}(\delta, y)\left(\delta-\delta_{0}\right)^{\chi-1} \mathrm{~d} \delta+\mathscr{W}_{y}(\delta, y) \phi_{\delta_{0}}(\delta, y)\left(\delta-\delta_{0}\right)^{\chi-1} \mathrm{~d} W(\delta),
\end{aligned}
$$

where

$$
\begin{aligned}
\mathscr{L}_{\chi}^{\delta_{0}} \mathscr{W}(\delta, y) & =\mathscr{W}_{\delta}(\delta, y)\left(\delta-\delta_{0}\right)^{1-\chi}+\mathscr{W}_{y}(\delta, y) h_{\delta_{0}}(\delta, y)+\frac{1}{2} \operatorname{trace}\left[\phi_{\delta_{0}}(\delta, y)^{\delta} \mathscr{W}_{y y}(\delta, y) \phi_{\delta_{0}}(\delta, y)\left(\delta-\delta_{0}\right)^{\chi-1}\right] \\
\mathscr{W}_{\delta}(\delta, y) & =\frac{\partial \mathscr{W}(\delta, y)}{\partial \delta}(\delta, y) \\
\mathscr{W}_{y}(\delta, y) & =\left(\frac{\partial \mathscr{W}(\delta, y)}{\partial y_{1}}(\delta, y), \ldots, \frac{\partial \mathscr{W}(\delta, y)}{\partial y_{n}}(\delta, y)\right) \\
\mathscr{W}_{y y}(\delta, y) & =\left(\frac{\partial^{2} \mathscr{W}(\delta, y)}{\partial y_{i} \partial y_{j}}(\delta, y)\right)_{n \times n} .
\end{aligned}
$$

Theorem 1. Let $p$ be a positive constant. Suppose that there exist positive constants $\alpha_{1}, \alpha_{2}$, and $\alpha_{3}$. Assume that there exist $\mathscr{W}(\delta, y) \in C^{1,2}\left(\left[\delta_{0},+\infty\left[\times \mathbb{R}^{n}, \mathbb{R}^{+}\right)\right.\right.$such that

(i) $\alpha_{1}\left|y_{1}\right|^{p} \leq \mathscr{W}(\delta, y) \leq \alpha_{2}\left|y_{1}\right|^{p}, \forall(\delta, y) \in\left[\delta_{0},+\infty\left[\times \mathbb{R}^{n}\right.\right.$

(ii) $\mathscr{L}_{\chi}^{\delta_{0}} \mathscr{W}(\delta, y(\delta)) \leq-$

$\alpha_{3}\left|y_{1}(\delta)\right|^{p}, \forall(\delta, y) \in\left[\left[\delta_{0},+\infty\left[\times \mathbb{R}^{n}\right.\right.\right.$

Then, system (7) is $y_{1}$-ESPM.

Proof. Let $y_{0} \neq 0$ be fixed and denoted by $y(\delta)$ the solution of system (7). For each $\left|y_{0}\right| \leq d$, the stopping time is denoted by $\sigma_{d}$ such that

$$
\sigma_{d}=\inf \left\{\delta \geq \delta_{0} ;|y(\delta)| \geq d\right\} .
$$


It is easy to see that $\sigma_{d} \longrightarrow \infty$ as $d \longrightarrow \infty$. By Itô's formula, we obtain for $\delta \geq \delta_{0}$

$$
\begin{aligned}
& \Xi_{\chi}\left(\frac{\alpha_{3}}{\alpha_{2}},\left(\delta \wedge \sigma_{d}-\delta_{0}\right)\right) \mathscr{W}\left(\delta \wedge \sigma_{d}, y\left(\delta \wedge \sigma_{d}\right)\right)=\mathscr{W}\left(\delta_{0}, y\left(\delta_{0}\right)\right) \\
& \quad+\int_{\delta_{0}}^{\delta \wedge \sigma_{d}} \Xi_{\chi}\left(\frac{\alpha_{3}}{\alpha_{2}},\left(l-\delta_{0}\right)\right)\left(l-\delta_{0}\right)^{\chi-1}\left[\frac{\alpha_{3}}{\alpha_{2}} \mathscr{W}(l, y(l))+\mathscr{L}_{\chi}^{\delta_{0}} \mathscr{W}(l, y(l))\right] \mathrm{d} l \\
& \quad+\int_{\delta_{0}}^{\delta \wedge \sigma_{d}} \Xi_{\chi}\left(\frac{\alpha_{3}}{\alpha_{2}},\left(l-\delta_{0}\right)\right)\left(l-\delta_{0}\right)^{\chi-1} \mathscr{W}_{y}(l, y(l)) \phi_{\delta_{0}}(l, y(l)) \mathrm{d} W(l) .
\end{aligned}
$$

Using that $M(\delta)=\int_{\delta_{0}}^{\delta} \Xi_{\chi}\left(\alpha_{3} / \alpha_{2}, \quad\left(l-\delta_{0}\right)\right)\left(l-\delta_{0}\right)^{\chi-1}$ $\mathscr{W}_{y}(l, y(l)) \phi_{\delta_{0}}(l, y(l)) \mathrm{d} W(l)$ is a local martingale with
$M\left(\delta_{0}\right)=0$ (see [23]) and taking the expectation on both sides of (16), we obtain

$$
\begin{aligned}
& E\left(\Xi_{\chi}\left(\frac{\alpha_{3}}{\alpha_{2}},\left(\delta \wedge \sigma_{d}-\delta_{0}\right)\right) \mathscr{W}\left(\delta \wedge \sigma_{d}, y\left(\delta \wedge \sigma_{d}\right)\right)\right)=\mathscr{W}\left(\delta_{0}, y\left(\delta_{0}\right)\right) \\
& \quad+E\left(\int_{\delta_{0}}^{\delta \wedge \sigma_{d}} \Xi_{\chi}\left(\frac{\alpha_{3}}{\alpha_{2}},\left(l-\delta_{0}\right)\right)\left(l-\delta_{0}\right)^{\chi-1}\left[\frac{\alpha_{3}}{\alpha_{2}} \mathscr{W}(l, y(l))+\mathscr{L}_{\chi}^{\delta_{0}} \mathscr{W}(l, y(l))\right] \mathrm{d} l\right) .
\end{aligned}
$$

By conditions (i) and (ii), one has

$$
\begin{aligned}
& \alpha_{1} E\left(\Xi_{\chi}\left(\frac{\alpha_{3}}{\alpha_{2}},\left(\delta \wedge \sigma_{d}-\delta_{0}\right)\right)\left|y_{1}\left(\delta \wedge \sigma_{d}\right)\right|^{p}\right) \\
& \leq E\left(\Xi_{\chi}\left(\frac{\alpha_{3}}{\alpha_{2}},\left(\delta \wedge \sigma_{d}-\delta_{0}\right)\right) \mathscr{W}\left(\delta \wedge \sigma_{d}, y\left(\delta \wedge \sigma_{d}\right)\right)\right) \leq \alpha_{2}\left|y\left(\delta_{0}\right)\right|^{p} .
\end{aligned}
$$

Letting $d \longrightarrow \infty$, then

$$
\alpha_{1} E\left(\Xi_{\chi}\left(\frac{\alpha_{3}}{\alpha_{2}},\left(\delta-\delta_{0}\right)\right)\left|y_{1}(\delta)\right|^{p}\right) \leq \alpha_{2}\left|y\left(\delta_{0}\right)\right|^{p} .
$$

Hence, we can derive that

$$
E\left|y_{1}(\delta)\right|^{p} \leq \frac{\alpha_{2}}{\alpha_{1}} \Xi_{\chi}\left(-\frac{\alpha_{3}}{\alpha_{2}},\left(\delta-\delta_{0}\right)\right)\left|y\left(\delta_{0}\right)\right|^{p} .
$$

Therefore, system (7) is $y_{1}$-ESPM.

Remark 4. It is necessary to give the relation between equation (20) and the $y_{1}$-ASES to make our result more interesting.
Theorem 2. Let $p>2 / 2 \chi-1$. Suppose that (20) holds and there is a positive constant $M$ such that for all $(\delta, y) \in\left[\delta_{0}, \infty\right) \times \mathbb{R}^{n}$

$$
E\left|h_{\delta_{0}}\left(\delta, y_{1}\right)\right|^{p}+E\left|\phi_{\delta_{0}}\left(\delta, y_{1}\right)\right|^{p} \leq M E\left|y_{1}(\delta)\right|^{p} .
$$

Then, system (7) is $y_{1}$-ASES.

Proof. Let $y_{0} \neq 0$ be fixed. It follows from (20) that there exists positive constants $\xi_{1}$ and $\xi_{2}$ such that

$$
E\left|y_{1}(\delta)\right|^{p} \leq \xi_{1}\left|y\left(\delta_{0}\right)\right|^{p} \Xi_{\chi}\left(-\xi_{2},\left(\delta-\delta_{0}\right)\right), \quad \forall \delta \geq \delta_{0} \geq 0 .
$$


From ([23], p. 178), for any $t_{1}, t_{2}, t_{3} \geq 0$, we have

$$
\left(t_{1}+t_{2}+t_{3}\right)^{p} \leq\left[3\left(t_{1} \vee t_{2} \vee t_{3}\right)\right]^{p} \leq 3^{p}\left(t_{1}^{p} \vee t_{2}^{p} \vee t_{3}^{p}\right) \leq 3^{p}\left(t_{1}^{p}+t_{2}^{p}+t_{3}^{p}\right)
$$

Let $d=1,2,3, \ldots$ By system (7), we can derive that, for $\delta_{0}+d-1 \leq \delta \leq \delta_{0}+d$,

$$
y_{1}(\delta)=y_{1}\left(\delta_{0}+d-1\right)+\int_{\delta_{0}+d-1}^{\delta} h_{\delta_{0}}\left(l, y_{1}(l)\right)\left(l-\delta_{0}\right)^{\chi-1} \mathrm{~d} l+\int_{\delta_{0}+d-1}^{\delta} \phi_{\delta_{0}}\left(l, y_{1}(l)\right)\left(l-\delta_{0}\right)^{\chi-1} \mathrm{~d} W(l)
$$

Then,

$$
|y(\delta)| \leq\left|y_{1}\left(\delta_{0}+d-1\right)\right|+\int_{\delta_{0}+d-1}^{\delta}\left|h_{\delta_{0}}\left(l, y_{1}(l)\right)\right|\left(l-\delta_{0}\right)^{\chi-1} \mathrm{~d} l+\left|\int_{\delta_{0}+d-1}^{\delta} \phi_{\delta_{0}}\left(l, y_{1}(l)\right)\left(l-\delta_{0}\right)^{\chi-1} \mathrm{~d} W(l)\right|
$$

Hence,

$$
\begin{gathered}
\left|y_{1}(\delta)\right|^{p} \leq 3^{p}\left|y_{1}\left(\delta_{0}+d-1\right)\right|^{p}+3^{p}\left(\int_{\delta_{0}+d-1}^{\delta}\left|h_{\delta_{0}}\left(l, y_{1}(l)\right)\right|\left(l-\delta_{0}\right)^{\chi-1} \mathrm{~d} l\right)^{p} \\
+3^{p}\left|\int_{\delta_{0}+d-1}^{\delta} \phi_{\delta_{0}}\left(l, y_{1}(l)\right)\left(l-\delta_{0}\right)^{\chi-1} \mathrm{~d} W(l)\right|^{p}
\end{gathered}
$$

Therefore,

$$
\begin{aligned}
& \sup _{\delta_{0}+d-1 \leq \delta \leq \delta_{0}+d}\left|y_{1}(\delta)\right|^{p} \leq 3^{p}\left|y_{1}\left(\delta_{0}+d-1\right)\right|^{p} \\
& +3^{p} E\left(\int_{\delta_{0}+d-1}^{\delta_{0}+d}\left|h_{\delta_{0}}\left(l, y_{1}(l)\right)\right|\left(l-\delta_{0}\right)^{\chi-1} \mathrm{~d} l\right)^{p} \\
& +3^{p} \sup _{\delta_{0}+d-1 \leq \delta \leq \delta_{0}+d}\left|\int_{\delta_{0}+d-1}^{\delta} \phi_{\delta_{0}}\left(l, y_{1}(l)\right)\left(l-\delta_{0}\right)^{\chi-1} \mathrm{~d} W(l)\right|^{p} .
\end{aligned}
$$

Taking the expectation of (27), we obtain 


$$
\begin{aligned}
E\left[\sup _{\delta_{0}+d-1 \leq \delta \leq \delta_{0}+d}\left|y_{1}(\delta)\right|^{p}\right] \leq & 3^{p} E\left|y_{1}\left(\delta_{0}+d-1\right)\right|^{p} \\
& +3^{p} E\left(\int_{\delta_{0}+d-1}^{\delta_{0}+d}\left|h_{\delta_{0}}\left(l, y_{1}(l)\right)\right|\left(l-\delta_{0}\right)^{\chi-1} \mathrm{~d} l\right)^{p} \\
& +3^{p} E\left(\sup _{\delta_{0}+d-1 \leq \delta \leq \delta_{0}+d}\left|\int_{\delta_{0}+d-1}^{\delta} \phi_{\delta_{0}}\left(l, y_{1}(l)\right)\left(l-\delta_{0}\right)^{\chi-1} \mathrm{~d} W(l)\right|^{p}\right) .
\end{aligned}
$$

Using (22), we have

\section{Define}

$\left.E\left|y_{1}\left(\delta_{0}+d-1\right)\right|^{p} \leq \xi_{1}\left|y\left(\delta_{0}\right)\right|^{p} \Xi_{\chi}\left(-\xi_{2}, d-1\right)\right)$.

$$
\begin{aligned}
& I_{1}=E\left(\int_{\delta_{0}+d-1}^{\delta_{0}+d}\left|h_{\delta_{0}}\left(l, y_{1}(l)\right)\right|\left(l-\delta_{0}\right)^{\chi-1} \mathrm{~d} l\right)^{p} \\
& I_{2}=E\left(\sup _{\delta_{0}+d-1 \leq \delta \leq \delta_{0}+d}\left|\int_{\delta_{0}+d-1}^{\delta} \phi_{\delta_{0}}\left(l, y_{1}(l)\right)\left(l-\delta_{0}\right)^{\chi-1} \mathrm{~d} W(l)\right|^{p}\right.
\end{aligned}
$$

In view of (21) and (22) and the Hölder inequality,

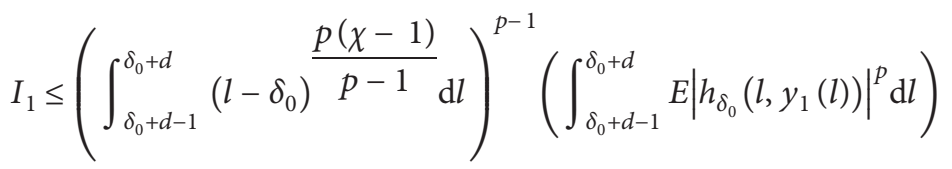

$$
\begin{aligned}
& \leq L_{1}^{p-1}\left(\int_{\delta_{0}+d-1}^{\delta_{0}+d} E\left|h_{\delta_{0}}\left(l, y_{1}(l)\right)\right|^{p} \mathrm{~d} l\right) \\
& \leq L_{1}^{p-1} M \int_{\delta_{0}+d-1}^{\delta_{0}+d} E\left|y_{1}(l)\right|^{p} \mathrm{~d} l \leq L_{1}^{p-1} M \xi_{1}\left|y\left(\delta_{0}\right)\right|^{p} \int_{\delta_{0}+d-1}^{\delta_{0}+d} \Xi_{\chi}\left(-\xi_{2},\left(\delta-\delta_{0}\right)\right) \mathrm{d} l \\
& \leq L_{1}^{p-1} M \xi_{1}\left|y\left(\delta_{0}\right)\right|^{p} \Xi_{\chi}\left(-\xi_{2}, d-1\right),
\end{aligned}
$$

where $L_{1}=p-1 / \chi p-1\left[d^{\chi p-1 / p-1}-(d-1)^{\chi p-1 / p-1}\right]$.

On the other hand, by (21) and (22), the Burkholder-Davis-Gundy inequality and Hölder inequality, we derive that 


$$
\begin{aligned}
I_{2} & \leq \Theta_{p} E\left(\int_{\delta_{0}+d-1}^{\delta_{0}+d}\left|\phi_{\delta_{0}}\left(l, y_{1}(l)\right)\right|^{2}\left(l-\delta_{0}\right)^{2(\chi-1)} \mathrm{d} l\right)^{p / 2} \\
& \leq \Theta_{p}\left(\int_{\delta_{0}+d-1}^{\delta_{0}+d}\left(l-\delta_{0}\right) \frac{2 p(\chi-1)}{p-2} \mathrm{~d} l\right)^{p-2 / 2} E\left(\int_{\delta_{0}+d-1}^{\delta_{0}+d}\left|\phi_{\delta_{0}}\left(l, y_{1}(l)\right)\right|^{p} \mathrm{~d} l\right) \\
& \leq \Theta_{p} L_{2}^{p-2 / 2} E\left(\int_{\delta_{0}+d-1}^{\delta_{0}+d}\left|\phi_{\delta_{0}}\left(l, y_{1}(l)\right)\right|^{p} \mathrm{~d} l\right) \\
& \leq \Theta_{p} L_{2}^{p-2 / 2} M \xi_{1}\left|y\left(\delta_{0}\right)\right|^{p} \Xi_{\chi}\left(-\xi_{2}, d-1\right),
\end{aligned}
$$

where $\quad L_{2}=p-2 / p(2 \chi-1)-2 \quad\left[d^{p(2 \chi-1)-2 / p-2}-(d-\right.$ 1) $\left.{ }^{p(2 \chi-1)-2 / p-2}\right]$ and $\Theta_{p}>0$ which only depends on $p$. Using (29) $-(33)$ and in view of (28),

$$
E\left[\sup _{\delta_{0}+d-1 \leq \delta \leq \delta_{0}+d}\left|y_{1}(\delta)\right|^{p}\right] \leq 3^{p} \xi_{1}\left|y\left(\delta_{0}\right)\right|^{p} \Xi_{\chi}\left(-\xi_{2}, d-1\right)\left[1+M\left(L_{1}^{p-1}+\Theta_{p} L_{2}^{p-2 / 2}\right)\right] .
$$

Let $v \in\left(0, \xi_{2}(\varepsilon)\right)$ be arbitrary, then Chebyshev theorem yields that

$$
\begin{aligned}
& \mathbb{P}\left\{\sup _{\delta_{0}+d-1 \leq \delta \leq \delta_{0}+d}\left|y_{1}(\delta)\right|^{p} \leq \xi_{1}\left|y\left(\delta_{0}\right)\right|^{p} \Xi_{\chi}\left(-\left(\xi_{2}-v\right), d\right)\right\} \\
& \leq \frac{\Xi_{\chi}\left(\xi_{2}-v, d\right)}{\xi_{1}\left|y\left(\delta_{0}\right)\right|^{p}} E\left[\sup _{\delta_{0}+d-1 \leq \delta \leq \delta_{0}+d}\left|y_{1}(\delta)\right|^{p}\right] \\
& \leq 3^{p} \Xi_{\chi}\left(\xi_{2}-v, d\right) \Xi_{\chi}\left(-\xi_{2}, d-1\right)\left[1+M\left(L_{1}^{p-1}+\Theta_{p} L_{2}^{p-2 / 2}\right)\right] .
\end{aligned}
$$

Set $V_{d}=\Xi_{\chi}\left(\xi_{2}-v, d\right) \Xi_{\chi}\left(-\xi_{2}, d-1\right)$. It is obvious to see that $V_{d} \sim \Xi_{\chi}(-v, d)$.

By the Borel-Cantelli lemma, there exists $d_{0}=d_{0}(\widetilde{\omega})$ such that for all $\widetilde{\omega} \in \widetilde{\Omega}$ and $d \geq d_{0}$, we obtain

$$
\sup _{\delta_{0}+d-1 \leq \delta \leq \delta_{0}+d}\left|y_{1}(\delta)\right|^{p} \leq \xi_{1}\left|y\left(\delta_{0}\right)\right|^{p} \Xi_{\chi}\left(-\left(\xi_{2}-v\right), d\right) \text {, a.s. }
$$

which implies that, for $\delta \in\left[\delta_{0}+d-1, \delta_{0}+d\right)$ and $d \in \mathbb{N}^{*}$,

$$
\left|y_{1}(\delta)\right|^{p} \leq \xi_{1}\left|y\left(\delta_{0}\right)\right|^{p} \Xi_{\chi}\left(-\left(\xi_{2}-v\right), d\right), \quad \text { a.s. }
$$

Letting $v \longrightarrow 0$, we deduce that, for any $\delta \in\left[\delta_{0}+d-\right.$ $\left.1, \delta_{0}+d\right)$ and $d \in \mathbb{N}^{*}$,

$$
\left|y_{1}(\delta)\right|^{p} \leq \xi_{1}\left|y\left(\delta_{0}\right)\right|^{p} \Xi_{\chi}\left(-\xi_{2},\left(\delta-\delta_{0}\right)\right), \quad \text { a.s. }
$$

which completes the proof.

Remark 5. We can see here the importance of Theorem 2 to make the relation between $y_{1}$-ESPM and $y_{1}$-ASES, which means that under condition (21) the $y_{1}$-ESPM implies $y_{1}$-ASES.

\section{Exponential Instability in the $p$-th Moment}

This section is devoted to presenting a sufficient condition that guarantees the exponential instability of system (7) in the $p$-th moment. The interest of this section is to provide the importance to prove the instability of system (7) in the $p$-th moment with respect to all variables before showing the partial stability in our example in Section 5. 
Definition 7. Let $p>0$. The solution $y(\delta)=\left(y_{1}(\delta), y_{2}(\delta)\right)$ of system (7) is called exponentially unstable in the $p$-th moment, if there exist positive numbers $\xi_{1}$ and $\xi_{2}$ satisfying, for any $y\left(\delta_{0}\right) \in \mathbb{R}^{n}$,

$E|y(\delta)|^{p} \geq \xi_{1}\left|y\left(\delta_{0}\right)\right|^{p} \Xi_{\chi}\left(-\xi_{2},\left(\delta-\delta_{0}\right)\right), \quad \forall \delta \geq \delta_{0} \geq 0$.

If $p=2$, system (7) is exponentially unstable in mean square.

Remark 6. In order to show the importance of Theorem 1, we cite the following theorem.
Theorem 3. Let $p$ be a positive constant. Suppose that there exist positive constants $\alpha_{1}, \alpha_{2}$, and $\alpha_{3}$. Assume that there exist $\mathscr{W}(\delta, y) \in C^{1,2}\left(\left[\delta_{0},+\infty\left[\times \mathbb{R}^{n}, \mathbb{R}^{+}\right)\right.\right.$such that

(i) $\alpha_{1}|y|^{p} \leq \mathscr{W}(\delta, y) \leq \alpha_{2}|y|^{p}, \forall(\delta, y) \in\left[\delta_{0},+\infty\left[\times \mathbb{R}^{n}\right.\right.$

(ii) $\mathscr{L}_{\chi}^{\delta_{0}} \mathscr{W}(\delta, y(\delta)) \geq-\alpha_{3}|y(\delta)|^{p}, \forall(\delta, y) \in\left[\delta_{0},+\infty\right.$ $\left[\times \mathbb{R}^{n}\right.$

Then, system (7) is exponentially unstable in the p-th moment.

Proof. Proceeding as the proof of Theorem 1, we have

$$
\begin{aligned}
& E\left(\Xi_{\chi}\left(\frac{\alpha_{3}}{\alpha_{1}},\left(\delta \wedge \sigma_{d}-\delta_{0}\right)\right) \mathscr{W}\left(\delta \wedge \sigma_{d}, y\left(\delta \wedge \sigma_{d}\right)\right)\right)=\mathscr{W}\left(\delta_{0}, y\left(\delta_{0}\right)\right) \\
& \quad+E\left(\int_{\delta_{0}}^{\delta \wedge \sigma_{d}} \Xi_{\chi}\left(\frac{\alpha_{3}}{\alpha_{1}},\left(l-\delta_{0}\right)\right)\left(l-\delta_{0}\right)^{\chi-1}\left[\frac{\alpha_{3}}{\alpha_{1}} \mathscr{W}(l, y(l))+\mathscr{L}_{\chi}^{\delta_{0}} \mathscr{W}(l, y(l))\right] \mathrm{d} l\right) .
\end{aligned}
$$

By conditions (i) and (ii), one has

$$
\begin{aligned}
& \alpha_{2} E\left(\Xi_{\chi}\left(\frac{\alpha_{3}}{\alpha_{1}},\left(\delta \wedge \sigma_{d}-\delta_{0}\right)\right)\left|y\left(\delta \wedge \sigma_{d}\right)\right|^{p}\right) \\
& \geq E\left(\Xi_{\chi}\left(\frac{\alpha_{3}}{\alpha_{1}},\left(\delta \wedge \sigma_{d}-\delta_{0}\right)\right) \mathscr{W}\left(\delta \wedge \sigma_{d}, y\left(\delta \wedge \sigma_{d}\right)\right)\right) \\
& \geq \alpha_{1}\left|y\left(\delta_{0}\right)\right|^{p} .
\end{aligned}
$$

Letting $d \longrightarrow \infty$, thus

$$
\alpha_{2} E\left(\Xi_{\chi}\left(\frac{\alpha_{3}}{\alpha_{1}},\left(\delta-\delta_{0}\right)\right)|y(\delta)|^{p}\right) \geq \alpha_{1}\left|y\left(\delta_{0}\right)\right|^{p} .
$$

Therefore, we can obtain

$$
E|y(\delta)|^{p} \geq \frac{\alpha_{1}}{\alpha_{2}} \Xi_{\chi}\left(-\frac{\alpha_{3}}{\alpha_{1}},\left(\delta-\delta_{0}\right)\right)\left|y\left(\delta_{0}\right)\right|^{p} .
$$

Finally, system (7) is exponentially unstable in the $p$-th moment.

Remark 7. The instability analysis of Theorem 3 is based on the Lyapunov function $\mathscr{W}(\delta, y)$. It is worth pointing out that the instability Theorem 3 can also handle the cases of conformable stochastic functional differential equations and conformable neutral stochastic functional differential equations.

\section{Example}

Consider the following CSS:

$$
\left\{\begin{array}{l}
T_{\delta_{0}}^{\chi} y_{1}(\delta)=h_{1}(\delta, y)+\phi_{1}(\delta, y) \frac{\mathrm{d} W_{1}(\delta)}{\mathrm{d} \delta} \\
T_{\delta_{0}}^{\chi} y_{2}(\delta)=h_{2}(\delta, y)+\phi_{2}(\delta, y) \frac{\mathrm{d} W_{2}(\delta)}{\mathrm{d} \delta}
\end{array}\right.
$$

where $\quad \chi \in(1 / 2,1], \quad y(\delta)=\left(y_{1}(\delta), y_{2}(\delta)\right) \in \mathbb{R}^{2}$, $y\left(\delta_{0}\right)=y_{0}=\left(y_{01}, y_{02}\right)$, and $\left\{W_{i}(\delta)\right\}_{i \in\{1,2\}}$ are one-dimensional Brownian motions. Let

$$
\begin{aligned}
& h_{1}(\delta, y)=-2 y_{1}(\delta)\left(1+y_{2}^{2}(\delta)\right), \\
& h_{2}(\delta, y)=y_{2}(\delta), \\
& \phi_{1}(\delta, y)=y_{1}(\delta) \sqrt{2\left(1+y_{2}^{2}(\delta)\right)}\left(\delta-\delta_{0}\right) \frac{1-\chi}{2}, \\
& \phi_{2}(\delta, y)=2 y_{1}(\delta) \sqrt{1+\frac{y_{2}^{2}(\delta)}{2}}\left(\delta-\delta_{0}\right) \frac{1-\chi}{2} .
\end{aligned}
$$

We will show that system (44) is exponentially unstable in mean square with respect to all variables.

Let $\mathscr{W}(\delta, y)=y_{1}^{2}+y_{2}^{2}$.

Thus,

$$
\left|y^{2}\right| \leq \mathscr{W}(\delta, y) \leq 2\left|y^{2}\right|
$$

$\mathscr{L}_{\chi}^{\delta_{0}} \mathscr{W}(\delta, y(\delta))=2\left(y_{1}^{2}(\delta)+y_{2}^{2}(\delta)\right) \geq-2|y(\delta)|^{2}$.

Consequently, by Theorem 3, system (44) is exponentially unstable in mean square.

Now, set $\mathscr{W}_{1}(\delta, y)=y_{1}^{2}$.

Then, 


$$
\begin{gathered}
y_{1}^{2} \leq \mathscr{W}(\delta, y) \leq 2 y_{1}^{2}, \\
\mathscr{L}_{\chi}^{\delta_{0}} \mathscr{W}(\delta, y(\delta))=-2 y_{1}^{2}(\delta)\left(1+y_{2}^{2}(\delta)\right) \leq-2 y_{1}^{2}(\delta) .
\end{gathered}
$$

Then, $\alpha_{1}=1, \alpha_{2}=\alpha_{3}=2$, and $p=2$. Therefore, all assumptions of Theorem 1 are satisfied. Therefore, system (44) is $y_{1}$-ESMS.

\section{Conclusion}

This paper investigated conformable stochastic systems which include the conformable fractional derivative and the Brownian motion.

Using the Lyapunov method and some classical stochastic calculus techniques like Itô's formula, we prove the ASES and the ESPM with respect to part of the variables of conformable stochastic systems. Finally, an example has been presented to show the interest of our results. Also, there are some pending questions to be solved in future work, for example, the partial stabilization of an unstable conformable neutral stochastic functional differential equations.

\section{Data Availability}

No data were used to support this study.

\section{Conflicts of Interest}

The authors declare that they have no conflicts of interest.

\section{Acknowledgments}

This work was funded by the Deanship of Scientific Research at Jouf University under Grant No. DSR-2021-03-0328.

\section{References}

[1] R. Khalil, M. Al Horani, A. Yousef, and M. Sababheh, "A new denition of fractional derivative," Journal of Computational and Applied Mathematics, vol. 264, p. 6570, 2014.

[2] S. Das, "Controllability of a class of conformable fractional differential system," Journal of Control and Decision, vol. 8, no. 4, pp. 415-421, 2020.

[3] O. Naifar, G. Rebiai, A. Ben Makhlouf, M. A. Hammami, and A. Guezane-Lakoud, "Stability analysis of conformable fractional-order nonlinear systems depending on a parameter," Journal of Applied Analysis, vol. 26, no. 2, pp. 287-296, 2020.

[4] A. Neirameh, "New fractional calculus and application to the fractional-order of extended biological population model," Boletim da Sociedade Paranaense de Matemática, vol. 36, no. 3, pp. 1-14, 2018.

[5] G. Xiao and J. Wang, "On the stability of solutions to conformable stochastic differential equations," Miskolc Mathematical Notes, vol. 21, no. 1, pp. 509-523, 2020.

[6] G. Xiao, J. Wang, and D. O’Regan, "Existence, uniqueness and continuous dependence of solutions to conformable stochastic differential equations," Chaos, Solitons \& Fractals, vol. 139, Article ID 110169, 2020.

[7] M. A. E. Abdelrahman and H. A. Alkhidhr, "Closed-form solutions to the conformable space-time fractional simplified $\mathrm{MCH}$ equation and time fractional Phi-4 equation," Results in Physics, vol. 18, Article ID 103294, 2020.
[8] D. Kumar, A. R. Seadawy, and A. K. Joardar, "Modified Kudryashov method via new exact solutions for some conformable fractional differential equations arising in mathematical biology," Chinese Journal of Physics, vol. 56, no. 1, pp. 75-85, 2018.

[9] M. Odabasi, "Exact analytical solutions of the fractional biological population model, fractional EW and modified EW equations," An International Journal of Optimization and Control: Theories \& Applications, vol. 11, no. 1, pp. 52-58, 2021.

[10] B. Ahmad, M. Alghanmi, A. Alsaedi, and R. P. Agarwal, "On an impulsive hybrid system of conformable fractional differential equations with boundary conditions," International Journal of Systems Science, vol. 51, no. 5, pp. 958-970, 2020.

[11] A. Souahi, O. Naifar, A. B. Makhlouf, and M. A. Hammami, "Discussion on Barbalat Lemma extensions for conformable fractional integrals," International Journal of Control, vol. 92, no. 2, pp. 234-241, 2019.

[12] A. Souahi, A. Ben Makhlouf, and M. A. Hammami, "Stability analysis of conformable fractional-order nonlinear systems," Indagationes Mathematicae, vol. 28, no. 6, pp. 1265-1274, 2017.

[13] A. Younas, T. Abdeljawad, R. Batool, A. Zehra, and M. A. Alqudah, "Linear conformable differential system and its controllability," Advances in Difference Equations, vol. 2020, Article ID 449, 2020.

[14] A. Younus, Z. Dastgeer, N. Ishaq, A. Ghaffar, K. S. Nisar, and D. Kumar, "On the observability of conformable linear timeinvariant control systems," Discrete \& Continuous Dynamical Systems - S, vol. 14, no. 10, p. 3837, 2021.

[15] T. Caraballo, L. Mchiri, and M. Rhaima, "Partial practical exponential stability of neutral stochastic functional differential equations with markovian switching," Mediterranean Journal of Mathematics, vol. 18, no. 4, p. 142, 2021.

[16] T. Caraballo, F. Ezzine, M. A. Hammami, and L. Mchiri, "Practical stability with respect to a part of variables of stochastic differential equations," Stochastics An International Journal of Probability and Stochastic Processes, vol. 93, no. 5, pp. 647-664, 2021.

[17] O. Ignatyev, "Partial asymptotic stability in probability of stochastic differential equations," Statistics \& Probability Letters, vol. 79, no. 5, pp. 597-601, 2009.

[18] D. Liu, W. Wang, O. Ignatyev, and W. Zhang, "Partial stochastic asymptotic stability of neutral stochastic functional differential equations with Markovian switching by boundary condition," Advances in Difference Equations, vol. 220, pp. 1-8, 2012.

[19] V. F. Sharov, "Partial stability and stabilization of stochastic systems," Avtomatika i telemechanika, vol. 11, pp. 63-71, 1978.

[20] V. I. Vorotnikov, Partial Stability and Control, Birkhauser, Boston, MA, USA, 1998.

[21] T. Abdeljawad, "On conformable fractional calculus," Journal of Computational and Applied Mathematics, vol. 279, pp. 57-66, 2015.

[22] M. Eslami, "Solitary wave solutions for perturbed nonlinear Schrodinger's equation with Kerr law nonlinearity under the DAM," Optik, vol. 126, no. 13, pp. 1312-1317, 2015.

[23] X. Mao, Stochastic Differential Equations and Applications, Ellis Horwood, Chichester, UK, 1997. 